\title{
Molecular characterization of group A rotaviruses detected in children with gastroenteritis in Ireland in 2006-2009
}

\author{
O. CASHMAN ${ }^{1}$, P. J. COLLINS ${ }^{1}$, G. LENNON ${ }^{1}$, B. CRYAN ${ }^{2}$, V. MARTELLA ${ }^{3}$, \\ S. FANNING ${ }^{4}$, A. STAINES ${ }^{5}$ AND H. O'SHEA ${ }^{1 *}$ \\ ${ }^{1}$ Molecular Epidemiology Laboratory, Cork Institute of Technology, Rossa Avenue, Bishopstown, Cork, Ireland \\ ${ }^{2}$ Department of Microbiology, Cork University Hospital, Wilton, Cork, Ireland \\ ${ }^{3}$ Department of Veterinary Public Health, Faculty of Veterinary Medicine of Bari, Valenzano, Bari, Italy \\ ${ }^{4}$ Centres for Food Safety \& Food-borne Zoonomics, UCD Veterinary Sciences Centre, School of Agriculture, \\ Food Science and Veterinary Medicine, University College Dublin, Belfield, Dublin, Ireland \\ ${ }^{5}$ Health Systems Research School of Nursing, Dublin City University, Dublin, Ireland
}

(Accepted 10 February 2011; first published online 14 March 2011)

\section{SUMMARY}

Community and hospital-acquired cases of human rotavirus are responsible for millions of gastroenteritis cases in children worldwide, chiefly in developing countries, and vaccines are now available. During surveillance activity for human rotavirus infections in Ireland, between 2006 and 2009, a total of 420 rotavirus strains were collected and analysed. Upon either PCR genotyping and sequence analysis, a variety of VP7 (G1-G4 and G9) and VP4 (P[4], P[6], P [8] and $\mathrm{P}[9])$ genotypes were detected. Strains G1P[8] were found to be predominant throughout the period 2006-2008, with slight fluctuations seen in the very limited samples available in 2008-2009. Upon either PCR genotyping and sequence analysis of selected strains, the G1, G3 and G9 viruses were found to contain E1 (Wa-like) NSP4 and I1 VP6 genotypes, while the analysed G2 strains possessed E2 NSP4 and I2 VP6 genotypes, a genetic make-up which is highly conserved in the major human rotavirus genogroups Wa- and Kun-like, respectively. Upon sequence analysis of the most common VP4 genotype, P[8], at least two distinct lineages were identified, both unrelated to P[8] Irish rotaviruses circulating in previous years, and more closely related to recent European humans rotaviruses. Moreover, sequence analysis of the VP7 of G1 rotaviruses revealed the onset of a G1 variant, previously unseen in the Irish population.

Key words: Epidemiology, Ireland, lineages, rotavirus.

\section{INTRODUCTION}

Group A rotaviruses are important human and animal enteric pathogens. Infection by group A rotavirus causes millions of childhood gastroenteritis cases worldwide and 600000 child deaths annually, chiefly

\footnotetext{
* Author for correspondence: Dr H. O'Shea, Molecular Epidemiology Laboratory, Cork Institute of Technology, Rossa Avenue, Bishopstown, Cork, Ireland. (Email: helen.oshea@cit.ie)
}

in developing countries [1]. Group A rotaviruses are classified antigenically and genetically, based on the main antigenic determinants, the outer capsid proteins VP7 and VP4, which specify the G and P serotypes/ genotypes, respectively [2]. At least $25 \mathrm{G}$ genotypes and $32 \mathrm{P}$ genotypes have been documented to date $[3,4]$. The major human $\mathrm{G}$ types are $\mathrm{G} 1, \mathrm{G} 2, \mathrm{G} 3, \mathrm{G} 4$, and $\mathrm{G} 9$ which, in combination with the $\mathrm{P}$ types $\mathrm{P}[8]$, $\mathrm{P}[4]$ and $\mathrm{P}[6]$, account for over $80 \%$ of rotavirusassociated gastroenteritis episodes worldwide $[5,6]$. 
A novel classification system has been adopted recently for classification of the 11 segments of the rotavirus genome [7]. This new system extends the traditional genotype-based system which made use of rotavirus gene segments encoding VP7 and VP4 proteins to all 11 rotavirus genome segments, applying nucleotide cut-off values, to distinguish genotypes. VP6, the inner capsid layer, was previously classified into four antigenic subgroups using monoclonal antibodies or into two major genogroups, based on sequence analysis [8]. The NSP4 is a non-structural protein involved in viral replication and possessing enterotoxic activity [9]. Based on amino-acid sequence comparison, the NSP4 was formerly classified into six genotypes, A-F [8]. Under the new classification system the VP6 and NSP4 genes are classified into at least $15 \mathrm{I}$ and $12 \mathrm{E}$ genotypes, respectively [3, 7, 10].

Rotavirus infection is more common in infants and young children but it can also occur in adults, especially those that are in close contact with young children, adult travellers, and institutionalized or hospitalized elderly patients [11].

Two rotavirus vaccines are currently available, RotaTeq and Rotarix. RotaTeq (Merck \& Co. Inc., USA) is a live, oral pentavalent human/bovine reassortant rotavirus vaccine [12-14], containing the more common human rotavirus (HRV) VP7 and VP4 antigens (G1, G2, G3, G4, and P[8]). Rotarix (GlaxoSmithKline Biologicals, Belgium), is a monovalent vaccine based on an attenuated human G1P[8] rotavirus strain $[15,16]$. Both vaccines have been proven to be safe and effective in protecting children against rotavirus disease [15-17]. Although Rotarix and Rotateq have been licensed in Ireland since 2006, their use has only been in the private market paediatric sector [18]. As the vaccine is not in widespread use it is difficult to elucidate the effect the vaccine will have on the rotavirus epidemiology in Ireland.

Group A rotaviruses are highly heterogenous genetically. Rotavirus evolution is a dynamic process, driven by various mechanisms, including accumulation of point mutations, reassortment, recombination and inter-species transmission [19]. The introduction of human rotavirus vaccines has raised the question of whether the vaccines can alter the epidemiology of these viruses. The strong population vaccine-derived immunity could trigger/enhance evolutive mechanisms already observed in other viruses [20] and select novel/unusual strains.

Epidemiological surveillance in the Republic of Ireland reported 2520 cases of acute gastroenteritis in
2007, of which 2326 (92\%) were associated with rotavirus infection. Children aged $0-4$ years appeared to be most affected ( $n=2255 / 2326,96.9 \%$ of cases), followed by children aged 5-9 years $(n=45 / 2326$, $1.9 \%)[21]$.

Rotavirus surveillance in the Republic of Ireland has been conducted almost uninterrupted since 1997 [22-24]. In this study, the distribution of human rotavirus $G$ and $P$ types in 2006-2009 was investigated. In addition, the genetic make up of selected strains was assessed in more detail by analysing the VP6 and NSP4 gene segments.

\section{MATERIALS AND METHODS}

\section{Specimen collection}

Rotavirus-positive faecal samples $(n=420)$ were collected regularly from October to September each year (2006-2007, $n=139 ; 2007-2008, n=268 ; 2008-2009$, $n=13$ ) from children aged $<5$ years from hospitals in the Munster region of Ireland. The hospitals included in the study were Cork University Hospital (CUH), Bon Secours Hospital, Cork, Mercy University Hospital, Cork, and Waterford Regional Hospital.

\section{Initial identification of rotavirus}

Rotavirus identification was performed in the hospitals' microbiology laboratories by use of immunochromatographic strips (Coris BioConcept, Belgium). To confirm the results obtained by the antigen detection method the genomic RNA was run and visualized on $1.5 \%(\mathrm{w} / \mathrm{v})$ agarose gel, which by virtue of the presence of the 11 dsRNA confirmed the presence of rotavirus.

\section{Nucleic acid extraction and analysis}

Viral RNA was extracted from the supernatant of $10 \%$ stool samples by SDS and proteinase K (SigmaAldrich, Ireland) digestion and phenol chloroform extraction, followed by precipitation with $100 \%$ ethanol overnight. Nucleic acid was resuspended in $100 \mu 1$ nuclease-free water and stored at $-80^{\circ} \mathrm{C}$, prior to use. The RNA extracts were examined by electrophoresis in $1.5 \%(\mathrm{w} / \mathrm{v})$ agarose gels and $10 \%(\mathrm{w} / \mathrm{v})$ polyacrylamide gels. Polyacrylamide gel electrophoresis (PAGE) was performed according to the Laemmli system [25], with a substitution of Long Ranger Gel solution (Cambrix Biosciences, UK) 
for acrylamide/bis. Electrophoresis was performed at $200 \mathrm{~V}$ for $5 \mathrm{~h}$ in $1 \times \mathrm{TBE}$ (Tris-borate EDTA). Following electrophoresis, the RNA bands were stained with ethidium bromide and visualized under UV light.

\section{Reverse transcriptase-polymerase chain reaction (RT-PCR)}

\section{$R T-P C R$ of $V P 7$ and $G$ genotyping}

Amplification and G genotyping of the VP7 gene was performed using RT-PCR and a nested multiplex PCR with type-specific primers (see Supplementary Table S1, available online) [26, 27]. Briefly, prior to RT-PCR, $3 \mu 1$ dsRNA was denatured in $3.5 \mu 1$ DMSO at $95^{\circ} \mathrm{C}$ for $5 \mathrm{~min}$. The RT-PCR reactions were performed in $50 \mu 1$ reaction volumes with the following reagents: $5 \mu \mathrm{l}$ of $10 \times$ reaction buffer, $1.5 \mu \mathrm{l}$ of $50 \mathrm{~mm} \mathrm{MgCl} 2$ (Euroclone, Life Sciences Division, Italy), $8 \mu \mathrm{l}$ dNTP mix (consisting of $1.25 \mathrm{~mm}$ each dNTP), $0.5 \mu 1$ of $40 \mathrm{U} / \mu 1$ ribonuclease inhibitor, $0 \cdot 2 \mu 1$ of $10 \mathrm{U} / \mu 1$ AMV-RT (Promega, USA), $0 \cdot 25 \mu 1$ of $5 \mathrm{U} / \mu \mathrm{l} \mathrm{Taq}$ polymerase (Euroclone), and $2 \mu \mathrm{l}$ of Beg 9 and End 9 primers (50 pmol) [26].

DNAse treatment with AMP-D1 DNAse 1 (SigmaAldrich Ireland Ltd) of samples was performed where necessary (i.e. samples which failed to generate a RT-PCR product on the first attempt were DNAse treated, to exclude any possible contamination of DNA from the RNA sample). All amplifications were performed in a Biometra T3000 thermocycler. RT-PCR protocol for the amplification of the VP7 gene consisted of $45^{\circ} \mathrm{C}$ for $30 \mathrm{~min}, 70^{\circ} \mathrm{C}$ for $4 \mathrm{~min}$, followed by 35 cycles of $94{ }^{\circ} \mathrm{C}$ for $1 \mathrm{~min}, 57^{\circ} \mathrm{C}$ for $30 \mathrm{~s}, 68^{\circ} \mathrm{C}$ for 2 min with a final extension time of $68{ }^{\circ} \mathrm{C}$ for $5 \mathrm{~min}$. G typing reactions consisted of $2 \mu \mathrm{l}$ of a 1:200 diluted VP7 RT-PCR product as the template for the subsequent typing reaction which consisted of $95^{\circ} \mathrm{C}$ for $5 \mathrm{~min}$, followed by 25 cycles of $95{ }^{\circ} \mathrm{C}$ for $1 \mathrm{~min} 52^{\circ} \mathrm{C}$ for $2 \mathrm{~min}, 72{ }^{\circ} \mathrm{C}$ for 2 min with a final extension step of $68^{\circ} \mathrm{C}$ for $5 \mathrm{~min}$.

Amplified products were resolved by conventional agarose gel electrophoresis $[1 \cdot 5 \%(\mathrm{w} / \mathrm{v})]$ at $100 \mathrm{~V}$ for $60 \mathrm{~min}$ in $1 \times \mathrm{TAE}$ buffer (Tris-acetate EDTA), stained with ethidium bromide and visualized by UV light.

\section{Amplification of VP4 and P genotyping}

Primers used in the amplification and $\mathrm{P}$ typing of the VP4 gene can be seen in Supplementary Table S1 (online) [28-30]. Thermal reactions and conditions similar to those described above were performed using the primer pairs Con2/Con3 and VP4F/VP4R allowing for the amplification of the VP4 gene at an annealing temperature of $52{ }^{\circ} \mathrm{C}$ for $30 \mathrm{~s}$. The seminested multiplex P typing reaction consisted of $2 \mu$ of a 1:100 diluted VP4 RT-PCR product as the template for the subsequent typing reactions which were: $95^{\circ} \mathrm{C}$ for $5 \mathrm{~min}$, followed by 25 cycles of $95^{\circ} \mathrm{C}$ for $1 \mathrm{~min}$ $54{ }^{\circ} \mathrm{C}$ for $2 \mathrm{~min}, 72^{\circ} \mathrm{C}$ for $2 \mathrm{~min}$ with a final extension step of $68^{\circ} \mathrm{C}$ for $5 \mathrm{~min}$.

\section{Amplification of VP6 and NSP4 and NSP4 genotyping}

The reverse-transcribed NSP4 PCR products were generated using 151/152 consensus primers [31], resulting in a 750-bp PCR product. A subsequent multiplex PCR assay was used to identify the three HRV major NSP4 genotypes E1-E3, previously known as Wa-like, KUN-like and AU1-like, respectively. This assay was performed under conditions previously described [31]. The amplicons were subjected to electrophoresis on agarose gels and stained with ethidium bromide. Amplification products of 647, 201 and $547 \mathrm{bp}$ indicated the presence of genotypes E1, E2 and E3, respectively.

VP6 gene segments were amplified as previously described [32] using the primer pair VP6F/VP6R at a annealing temperature of $54{ }^{\circ} \mathrm{C}$ for $30 \mathrm{~s}$, the resulting RT-PCR products were subjected to sequence analysis to determine the typing profile of VP6 gene segments of differing $G$ and $P$ types. Primers used in the amplification of VP6 and NSP4 products can be seen in Supplementary Table S2 (online).

\section{Nucleotide sequencing and phylogenetic analysis}

Subsets of all RT-PCR products from samples representing each type were selected for sequencing. The first-round PCR products were purified using a QIAquick PCR purification kit (Qiagen Ltd, UK), and sequenced commercially by Eurofins MWG Operon (MWG-biotech, Germany). Evaluation of all sequencing data was performed initially using DNAStar software (www.dnastar.com), and compared to those in the current GenBank database using BLAST analysis. Subsequent analysis was performed using Clustal W alignment (www.ebi.ac.uk/ clustalw), and Mega4.0 (www.megasoftware.net) for phylogentic tree construction. Accession numbers representing the different gene segments analysed and sequences submitted to Genbank are VP7 
(G types): HM126593-HM126600, HM560972 (ranging in size from 724 bp to $921 \mathrm{bp}$ ); VP4 (P types): HM126602-HM126605 (561-798 bp), HQ667789HQ667791 (560-561 bp); NSP4 (E types): HM137003-HM137011 (597-653 bp with one sample at $418 \mathrm{bp}$ ), and VP6 (I types): HM137012-HM137018 (255-322 bp).

\section{RESULTS}

\section{PAGE}

A total of 110 samples were examined using PAGE. The resulting electrophorotypes were visualized under UV light using the DNR Bio Imaging system. For all viruses, the banding pattern was in a 4, 2, 3, 2 formation, which is characteristic of group A rotavirus.

\section{G and $P$ typing}

Of the 420 samples collected from the selected hospitals in the Munster region, 306 were G-typed and 244 were successfully P-typed. A total of 215 samples were successfully characterized in both the VP4 and VP7 genes (Table 1). To confirm the accuracy of the typing assays, the sequence of selected strains of differing $G$ and $\mathrm{P}$ types were determined and analysed using BLAST (http://blast.ncbi.nlm.nih.gov/Blast.cgi). The most widely detected rotavirus strain types identified were G1P[8] $(n=153 / 215,71 \cdot 2 \%), \mathrm{G} 3 \mathrm{P}[8](n=26 /$ $215,12 \cdot 1 \%$ ) and G2P[4] and G9P[8] with an equal number of infections $(n=9 / 215,4 \cdot 2 \%)$, mixed infections of $\mathrm{G} 1+\mathrm{G} 3 \mathrm{P}[8]$ accounted for $n=7 / 215(3 \cdot 3 \%)$ (Table 1). The majority of G2P[4] and G1+ $\mathrm{G} 2 \mathrm{P}[4+8]$ infections identified over the 3 years were found in the first year (2006) of the study. While no G4 single infection was detected in the Irish population, mixed infections involving $\mathrm{G} 1+\mathrm{G} 3+\mathrm{G} 4 \mathrm{P}[8]$ were identified in two samples over the 2006-2009 study period (Table 1). In total, eight VP7 sequences, representing $\mathrm{G}$ types 1,2,3, and 9 were used to create phylogenetic trees seen in Figures 1-3†.

$\dagger$ In Figures 1-6 evolutionary relationships of VP7, VP6, VP4 and NSP4 sequences were inferred using the Neighbour-Joining method. The bootstrap consensus tree inferred from 100 replicates is taken to represent the evolutionary history of the taxa analysed. Branches corresponding to partitions reproduced in $<70 \%$ bootstrap replicates are collapsed. Irish sequences are in bold. Figures 1, 2 and 3 represent the VP7 sequences analysed, with each tree outlining the different lineages of each sequence. Figure 4 represents VP4 P[8] sequences, and Figures 5 and 6 outline NSP4 and VP6 sequences, respectively. Phylogenetic analyses were conducted in Mega4.0 [55].
Within G1 samples, Irish sequences were found to cluster within lineages I and II (Fig. 1). Within lineage I, Irish samples were previously found to cluster within sublineage Ic [22], this report identifies for the first time an Irish isolate (CIT-H57) that clusters within sublineage Ia.

The Irish G3 strains appeared to be less heterogeneous, as all G3 VP7 sequences were found to cluster within G3 lineage I. The Irish G9 sample CIT-H382 was found to cluster within lineage IIId (Figs 2, 3).

The predominant $\mathrm{P}$ type found during the course of this study, in combination with both single and mixed G types was P $[8](n=200 / 215,93 \cdot 0 \%)$. Other P types found included $\mathrm{P}[4](n=9 / 215,4 \cdot 2 \%) \mathrm{P}[6](n=1 / 215$, $0 \cdot 5 \%), \mathrm{P}[8+9](n=1 / 215,0 \cdot 5 \%) \mathrm{P}[4+8](n=4 / 215$, $1.9 \%$ ) (Table1). Upon sequence analysis, three $\mathrm{P}[8]$ strains (CIT-H190, CIT-H247, CIT-H282) were shown to cluster within lineage III (Fig. 4). Although previous strains identified from the same region of Ireland (R114, R115 and R386, isolated between 2003 and 2006) also cluster within lineage III, the recent Irish strains CIT-H190, CIT-H247 and CIT-H282 were found to cluster more closely with $\mathrm{P}[8]$ strains isolated from Italy and Russia. Another strain, CIT$\mathrm{H} 245$, clustered within lineage IV (Fig. 4). This lineage had not previously been shown to be present in circulating strains within Ireland [22-24].

Analysis of $\mathrm{P}$ [4] strains was initially hampered slightly by mistyping of the degenerate genotyping primer $\mathrm{P}[8]$ to an area of complementarity in the 3 , end of $\mathrm{P}[4]$ isolates, yielding a product size similar to $\mathrm{P}[8]$ isolates (P[8] 245 bp instead of $\mathrm{P}[4] 378 \mathrm{bp}$ ). As $\mathrm{G} 2$ isolates are commonly found in combination with $\mathrm{P}[4]$ and not $\mathrm{P}[8]$, all isolates were re-examined using sequencing analysis, a small representative proportion of which can be seen in Figure 4. Other P types identified in this study include P[6] and P[9], with no evidence of mistyping detected.

\section{NSP4 and VP6 analysis}

NSP4 analysis was performed on 103 samples, selected based on each $\mathrm{G}$ type detected. Of the 103 samples selected, 92 samples were typed as E1, and 11 samples were typed as E2. None of the samples were typed as E3. Of the 92 samples subjected to typing, 17 were selected for sequence analysis based on their NSP4 typing characteristics, from this, nine representative sequences were selected for inclusion in the phylogenetic tree (Fig. 5). The E- and G-type combinations identified were in agreement with previously reported 
Table 1. Distribution of G- and P-type combinations circulating in Ireland, from 2006-2009

\begin{tabular}{|c|c|c|c|c|c|c|c|c|}
\hline & \multicolumn{2}{|c|}{$\begin{array}{l}\text { 2006-2007 samples } \\
\text { collected }(n=139)\end{array}$} & \multicolumn{2}{|c|}{$\begin{array}{l}\text { 2007-2008 samples } \\
\text { collected }(n=268)\end{array}$} & \multicolumn{2}{|c|}{$\begin{array}{l}\text { 2008-2009 samples } \\
\text { collected }(n=13)\end{array}$} & \multicolumn{2}{|c|}{ Total } \\
\hline & $n$ & $\%$ & $n$ & $\%$ & $n$ & $\%$ & $n$ & $\%$ \\
\hline G1P[8] & 52 & $66 \cdot 7$ & 98 & $76 \cdot 6$ & 3 & $33 \cdot 3$ & 153 & $71 \cdot 2$ \\
\hline G2P[4] & 6 & $7 \cdot 7$ & 2 & $1 \cdot 6$ & 1 & $11 \cdot 1$ & 9 & $4 \cdot 2$ \\
\hline G3P[6] & 0 & $0 \cdot 0$ & 1 & $0 \cdot 8$ & 0 & $0 \cdot 0$ & 1 & 0.5 \\
\hline G3P[8] & 10 & $12 \cdot 8$ & 14 & $10 \cdot 9$ & 2 & $22 \cdot 2$ & 26 & $12 \cdot 1$ \\
\hline G4P[8] & 0 & $0 \cdot 0$ & 0 & $0 \cdot 0$ & 0 & $0 \cdot 0$ & 0 & $0 \cdot 0$ \\
\hline G9P[8] & 2 & $2 \cdot 6$ & 7 & $5 \cdot 5$ & 0 & $0 \cdot 0$ & 9 & $4 \cdot 2$ \\
\hline G1G2P[4 + 8] & 3 & $3 \cdot 8$ & 0 & $0 \cdot 0$ & 0 & $0 \cdot 0$ & 3 & $1 \cdot 4$ \\
\hline G1G3P[8] & 1 & $1 \cdot 3$ & 3 & $2 \cdot 3$ & 3 & $33 \cdot 3$ & 7 & $3 \cdot 3$ \\
\hline G1G3G4P[8] & 1 & $1 \cdot 3$ & 1 & $0 \cdot 8$ & 0 & $0 \cdot 0$ & 2 & $0 \cdot 9$ \\
\hline G1G9P[8] & 2 & $2 \cdot 6$ & 0 & $0 \cdot 0$ & 0 & $0 \cdot 0$ & 2 & $0 \cdot 9$ \\
\hline G1G9P[8 + 9] & 1 & $1 \cdot 3$ & 0 & $0 \cdot 0$ & 0 & $0 \cdot 0$ & 1 & $0 \cdot 5$ \\
\hline G1G3G9P[8] & 0 & $0 \cdot 0$ & 1 & $0 \cdot 8$ & 0 & $0 \cdot 0$ & 1 & $0 \cdot 5$ \\
\hline \multirow[t]{2}{*}{$\mathrm{G} 2 \mathrm{G} 3 \mathrm{P}[4+8]$} & 0 & $0 \cdot 0$ & 1 & $0 \cdot 8$ & 0 & $0 \cdot 0$ & 1 & $0 \cdot 5$ \\
\hline & 78 & 100 & 128 & 100 & 9 & 100 & 215 & 100 \\
\hline \multicolumn{9}{|c|}{ Individual $\mathbf{G}$ types detected out of $\mathbf{4 2 0}$ samples collected } \\
\hline G1 & 67 & $48 \cdot 2$ & 137 & $51 \cdot 1$ & 3 & $23 \cdot 1$ & 207 & $49 \cdot 3$ \\
\hline G2 & 8 & $5 \cdot 8$ & 3 & $1 \cdot 1$ & 2 & $15 \cdot 4$ & 13 & $3 \cdot 1$ \\
\hline G3 & 16 & $11 \cdot 5$ & 23 & $8 \cdot 6$ & 2 & $15 \cdot 4$ & 41 & $9 \cdot 8$ \\
\hline G9 & 2 & $1 \cdot 4$ & 13 & $4 \cdot 9$ & 0 & $0 \cdot 0$ & 15 & $3 \cdot 6$ \\
\hline $\mathrm{G} 1+2$ & 3 & $2 \cdot 2$ & 1 & $0 \cdot 4$ & 0 & $0 \cdot 0$ & 4 & $1 \cdot 0$ \\
\hline $\mathrm{G} 1+3$ & 2 & $1 \cdot 4$ & 9 & $3 \cdot 4$ & 3 & $23 \cdot 1$ & 14 & $3 \cdot 3$ \\
\hline $\mathrm{G} 1+9$ & 3 & $2 \cdot 2$ & 2 & $0 \cdot 7$ & 0 & $0 \cdot 0$ & 5 & $1 \cdot 2$ \\
\hline $\mathrm{G} 1+3+4$ & 1 & $0 \cdot 7$ & 2 & $0 \cdot 7$ & 0 & $0 \cdot 0$ & 3 & $0 \cdot 7$ \\
\hline $\mathrm{G} 1+3+9$ & 0 & $0 \cdot 0$ & 2 & $0 \cdot 7$ & 0 & $0 \cdot 0$ & 2 & $0 \cdot 5$ \\
\hline $\mathrm{G} 2+3$ & 0 & $0 \cdot 0$ & 0 & $0 \cdot 0$ & 1 & $7 \cdot 7$ & 1 & $0 \cdot 2$ \\
\hline $\mathrm{G} 2+9$ & 0 & $0 \cdot 0$ & 1 & $0 \cdot 4$ & 0 & $0 \cdot 0$ & 1 & $0 \cdot 2$ \\
\hline G typing total & 102 & $73 \cdot 4$ & 193 & $72 \cdot 0$ & 11 & $84 \cdot 6$ & 306 & $72 \cdot 9$ \\
\hline \multicolumn{9}{|c|}{ Individual $P$ types detected out of $\mathbf{4 2 0}$ samples collected } \\
\hline $\mathrm{P}[4]$ & 6 & $4 \cdot 3$ & 2 & $0 \cdot 7$ & 1 & $7 \cdot 7$ & 9 & $2 \cdot 1$ \\
\hline$P[6]$ & 0 & $0 \cdot 0$ & 1 & $0 \cdot 4$ & 0 & $0 \cdot 0$ & 1 & $0 \cdot 2$ \\
\hline $\mathrm{P}[8]$ & 78 & $56 \cdot 1$ & 142 & $53 \cdot 0$ & 9 & $69 \cdot 2$ & 229 & $54 \cdot 5$ \\
\hline $\mathrm{P}[4+8]$ & 3 & $2 \cdot 2$ & 1 & $0 \cdot 4$ & 0 & $0 \cdot 0$ & 4 & $1 \cdot 0$ \\
\hline $\mathrm{P}[8+9]$ & 1 & $0 \cdot 7$ & 0 & $0 \cdot 0$ & 0 & $0 \cdot 0$ & 1 & $0 \cdot 2$ \\
\hline P typing total & 88 & $63 \cdot 3$ & 146 & $54 \cdot 5$ & 10 & $76 \cdot 9$ & 244 & $58 \cdot 0$ \\
\hline
\end{tabular}

type combinations [31], in which G2 typing strains were found to have an E2 NSP4 profile, while G1, G3 and G9 strains displayed an E1 NSP4 profile.

Analysis of the VP6 gene of rotavirus was also performed on selected samples of differing $G$ types. A total of 11 samples comprising of four G1, one G9, two G3 and four G2 genotypes were analysed as previously described [31]. The G1, G3 and G9 samples clustered into the VP6 I1 genotype, while the G2P[4] samples clustered within VP6 I2. The corresponding NSP4 typing profile identified samples with a I1 VP6 profile to have a E1 NSP4 profile and those with I2 VP6 to have a E2 NSP4 profile (Figs 5, 6).

\section{DISCUSSION}

Rotavirus is responsible for over 100 million cases of gastroenteritis worldwide, with over 600000 deaths occurring annually $[1,33]$. The predominant human G and $P$ types circulating worldwide are G1-G4, G9 and $\mathrm{P}[8], \mathrm{P}[4], \mathrm{P}[6]$, with the majority of rotavirus cases reporting G1P[8] as the dominant strain. Over the past few years, new virus genotypes have emerged and become dominant types in certain parts of the world, e.g. G12 was first detected in the Philippines in 1988 [34]. Since then, its global coverage has increased and G12 has been detected in various parts of Asia, 


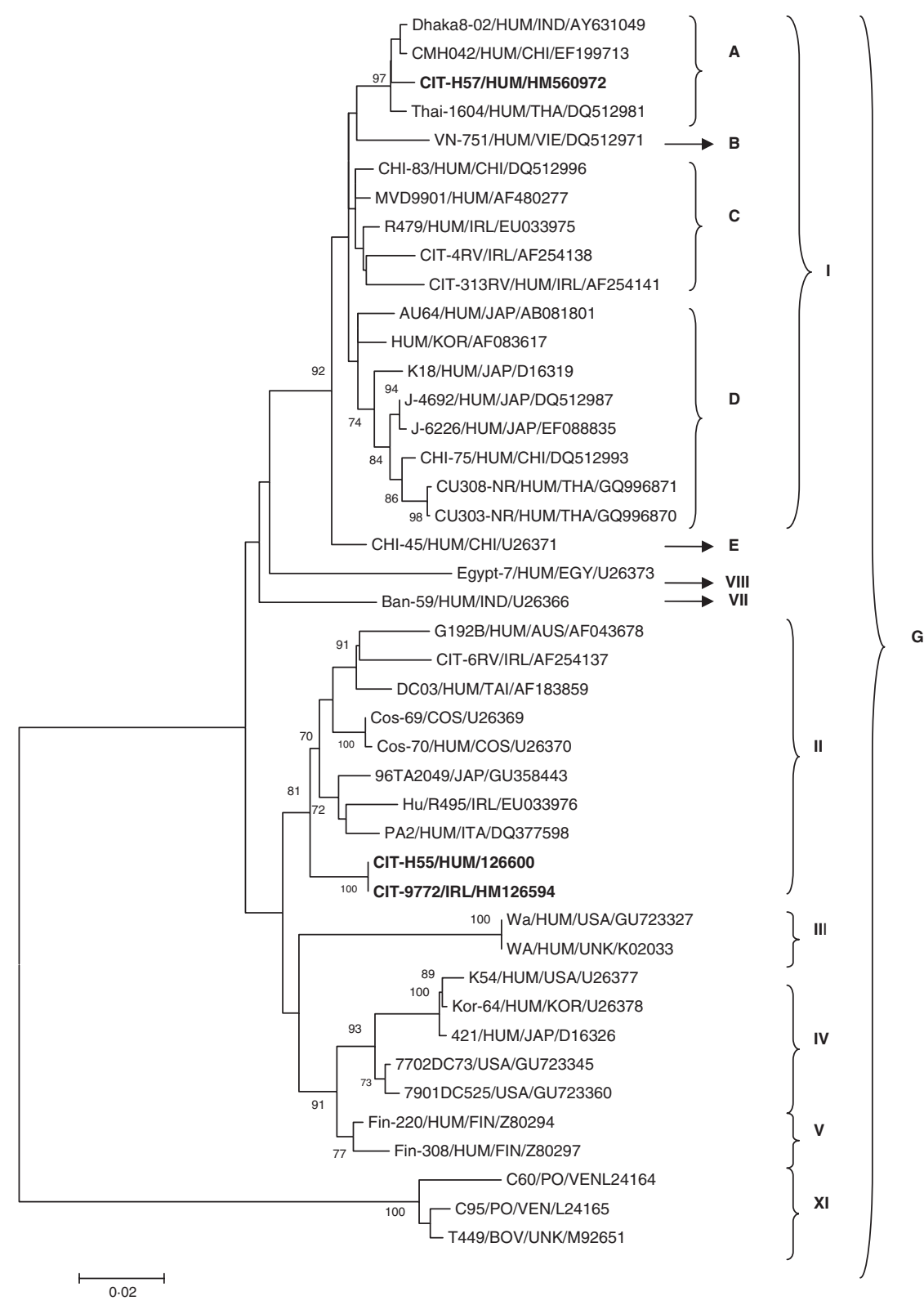

Fig. 1. Phylogenetic analysis of G1 nucleotide sequences, constructed using Clustal W alignment program and Mega4.0 (lineages adapted from [56]).

North and South America, as well as some parts of Europe [35-39]. It has become one of the most widely detected G types in India, along with G1 and G2 [27]. Similarly, G9 emerged as a dominant $G$ type both in Ireland [23] and worldwide, [40-43] and is now considered to be the fifth most dominant strain detected in humans. In Ireland, G9 peaked in the 2001-2002 season ( $n=25 / 83,30 \%)$ [23] and is still commonly detected in samples, albeit at much lower rates, with only 12 isolates $(n=12 / 288,3.6 \%)$ detected in 2003-2006 [24], and nine $(n=9 / 215,4 \cdot 2 \%)$ in the current study (2006-2009).
The main genotypes identified in this study were G1P[8] $(n=153 / 215,71 \cdot 2 \%)$ G3P[8] $\quad(n=26 / 215$, $12 \cdot 1 \%) \mathrm{G} 2 \mathrm{P}[4]$ and G9P[8], the latter were detected with an equal number of infections $(n=9 / 215,4 \cdot 2 \%)$, followed by $\mathrm{G} 1+\mathrm{G} 3$ mixed infection $(n=7 / 215$, $3 \cdot 3 \%$ ). The majority of $\mathrm{G} 2$ samples were detected in the first year of the study with $(n=6)$, followed by a reduced detection rate of $n=2$ and $n=1$ in the second and third years of the study, respectively (Table 1). Moreover, the common genotype G4 was not detected as a single infection, but was found only in combination with $\mathrm{G} 1+\mathrm{G} 3 \mathrm{P}[8]$ in two mixed infection 


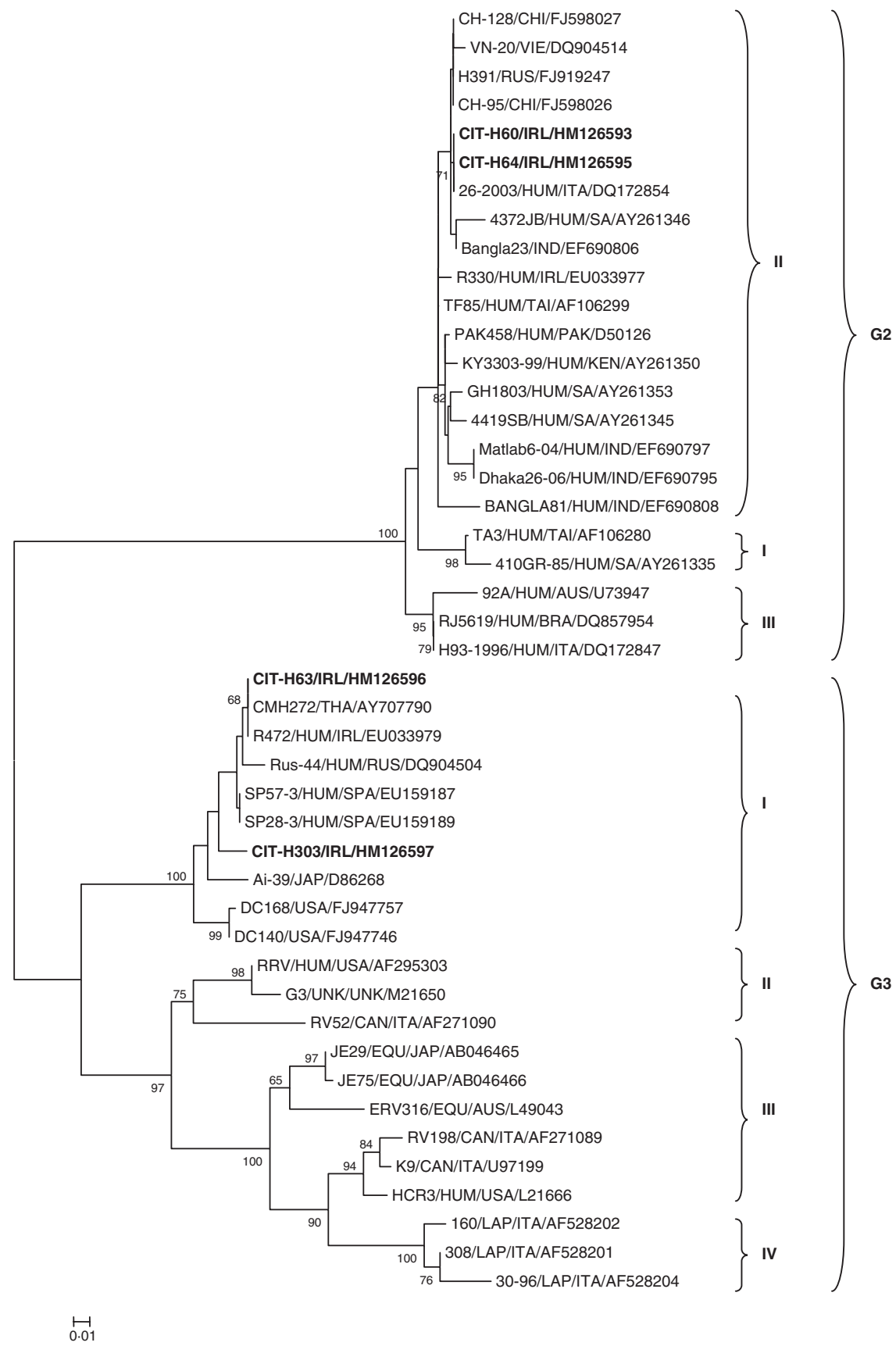

Fig. 2. Phylogenetic analysis of G2 and G3 nucleotide sequences, constructed using Clustal W alignment program and Mega4.0 (lineages adapted from [57]).

samples. This lack, or limited detection of G4 strains has been seen elsewhere in studies performed in Northern Ireland [44], Spain [45] and China [46]. The epidemiological data gathered during this surveillance period differs slightly from a pan-European study in which 16 countries identified G1P[8], G9P[8] and G2P[4] as the predominating strains in 2006-2007, followed by G1P[8], G4P[8] and G9P[8] in 2007-2008 and G1P[8] and G4P[8] in 2008-2009 [47].
Over the course of the study period, the primer pair Con2/Con3 [28] was found to lack sensitivity for the amplification of the VP4 gene product. In order to overcome this lack of sensitivity, a second primer set, VP4F/VP4R [29], was included in the amplification of the VP4 segment. The increased sensitivity of the VP4F/VP4R primer pair is thought to be due to a higher level of sensitivity to a wider diversity of rotavirus strains [29]. The originally designed Con $2 / \operatorname{Con} 3$ 


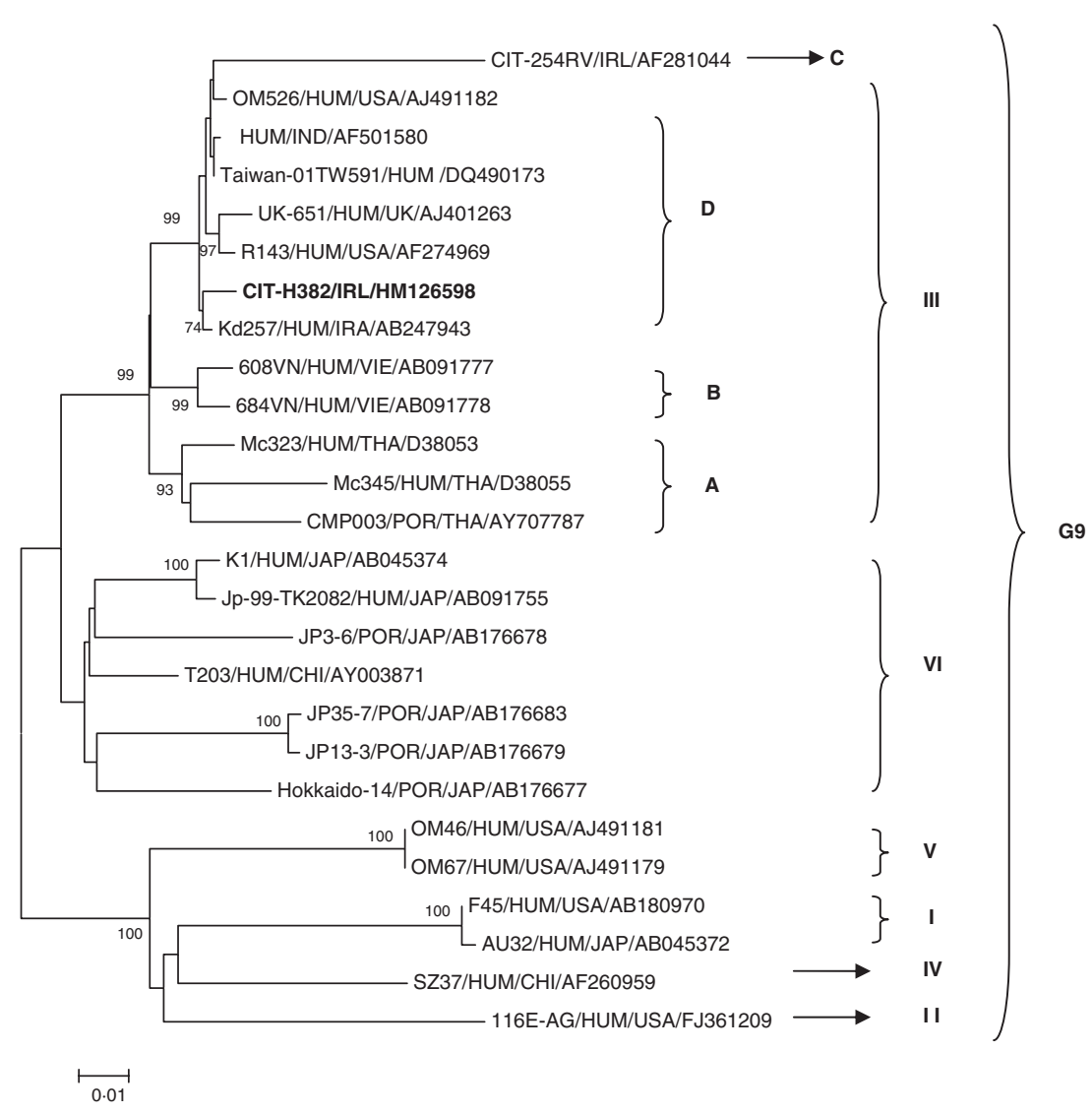

Fig. 3. Phylogenetic analysis of $\mathrm{G} 9$ nucleotide sequences, constructed using Clustal $\mathrm{W}$ alignment program and Mega4.0 (lineages adapted from [58]).

primer pair was derived from a limited number of cell culture-adapted strains available in public databases at the time of their design. The newer VP4F/VP4R primers were designed through analysis of the VP4 gene, for regions of consensus derived from over 200 different human P-typing strains of rotavirus in the last decade [29].

The majority of the samples for which the VP4 gene segment was successfully amplified and typed were $\mathrm{P}[8](n=200 / 215,93 \cdot 0 \%), \mathrm{P}[6](n=1 / 215,0 \cdot 5 \%)$ and $\mathrm{P}[8+9] \quad(n=1 / 215,0.5 \%)$ mixed infection; these samples were collected over a 3-year period (2006-2009). Single and mixed infection P[4] isolates $(n=13 / 215,6 \cdot 0 \%)$ were initially typed as $\mathrm{P}[8]$, due to an area of complementarity in the 3 ' end of the $\mathrm{P}[8]$ degenerate primer to $\mathrm{P}[4]$ isolates, as $\mathrm{G} 2$ isolates are rarely seen in combination with $\mathrm{P}[8]$ genotypes, all $\mathrm{P}$ types with a G2 genotype were subjected to sequence analysis which confirmed the presence of $\mathrm{P}[4]$ isolates in Irish samples, all $\mathrm{P}[4]$ isolates were identified in lineage V (Fig. 4).

Sequence analysis of $\mathrm{P}[8]$ isolates revealed that $3 / 4$ samples (CIT-H190, CIT-H247, CIT-H282) were shown to be within lineage III, with the remaining sample (CIT-H245) clustering within lineage IV (Fig. 4). Lineage IV strains also referred to as OP354like strains or $\mathrm{P}[8]$ sublineage $\mathrm{b}$ were first identified in Malawi in 1999 [48]. Since then it has been detected in various countries around the world including India [49], Thailand [50], Vietnam [51], Bangladesh [52] and now Ireland, suggesting widespread distribution around the world. Although incidence rates of this lineage are low in number, its continued presence in the global rotavirus population, may have an interesting effect on the efficacy of the rotavirus vaccines which contain the more commonly found $\mathrm{P}[8]$ genotypes and not lineage IV [53].

The emergence of new lineages and sublineages within Irish rotavirus strains and the expansion of strain diversity within previously identified G1P[8] lineages suggests that rotaviruses are continuously expanding their geographic range, which may offer an explanation as to its global dominance. A similar shift in the emergence of lineages previously unseen in the Irish bovine population has also been reported [54]. 


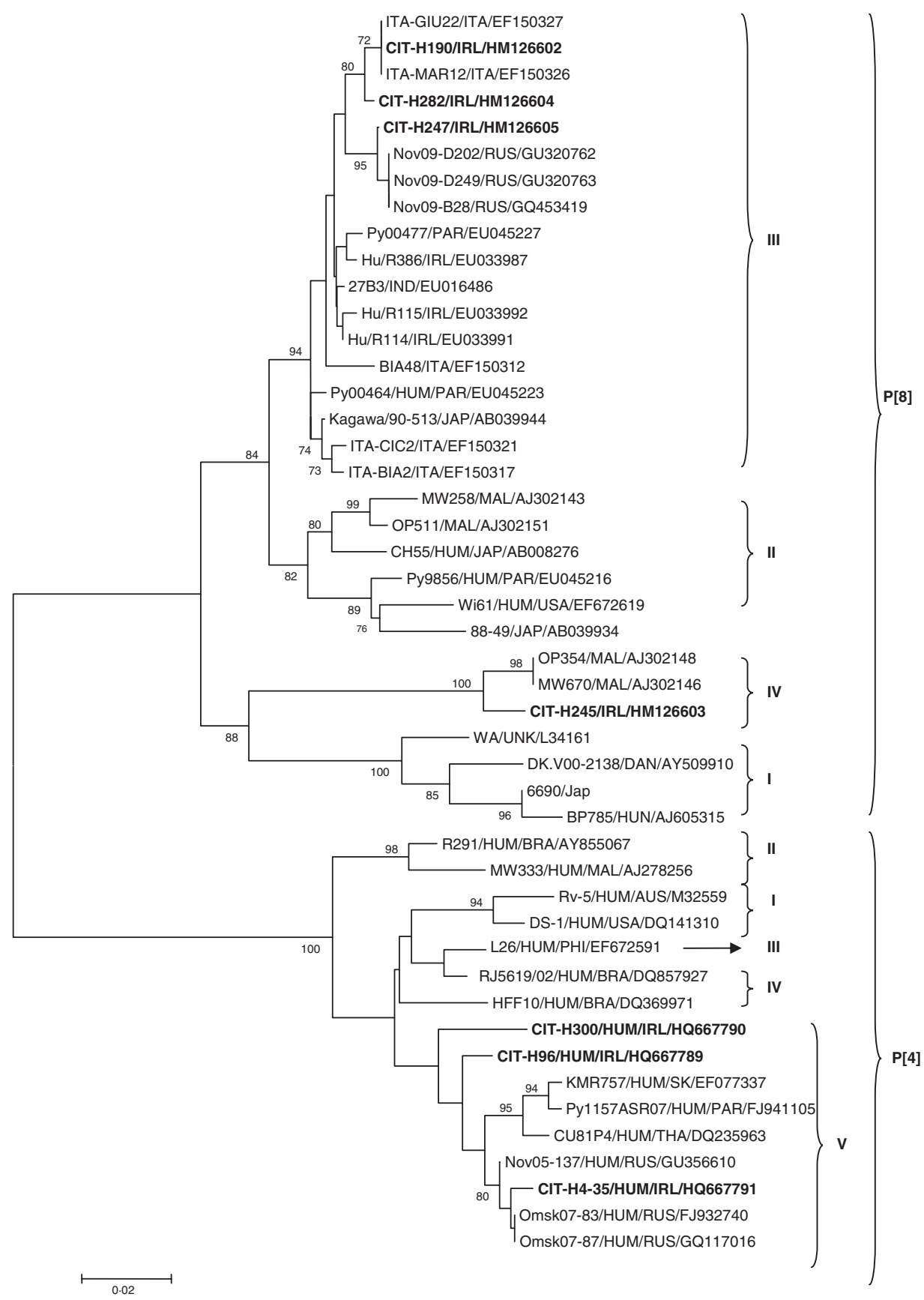

Fig. 4. Phylogenetic analysis of VP4 nucleotide sequences, constructed using Clustal W alignment program and Mega4.0 (lineages adapted from [59]).

NSP4 genotyping analysis was performed on 103 samples, representing every G type found in the study; all samples were found to be either E1, or E2, with no E3 strains being identified. The results of the NSP4 typing were in accordance with trends seen elsewhere [31], where strains with a G2P[4] genotype were found to have an E2 (NSP4), I2 (VP6) profile and strains with a G1P[8], G3P[8] and G9P[8] genotype demonstrated an E1,I1 profile. Interestingly, BLAST analysis performed on the partial VP6 region of CITH60 and CIT-H64 indicated a possible reassortment event between bovine sequences which can be seen in Figure 6, suggesting the need for further surveillance and genomic analysis to determine the possibility and the extent of the reassortment between human and bovine rotavirus.

This study reports on rotavirus $\mathrm{G}$ and $\mathrm{P}$ types circulating in Ireland between 2006 and 2009. Results 


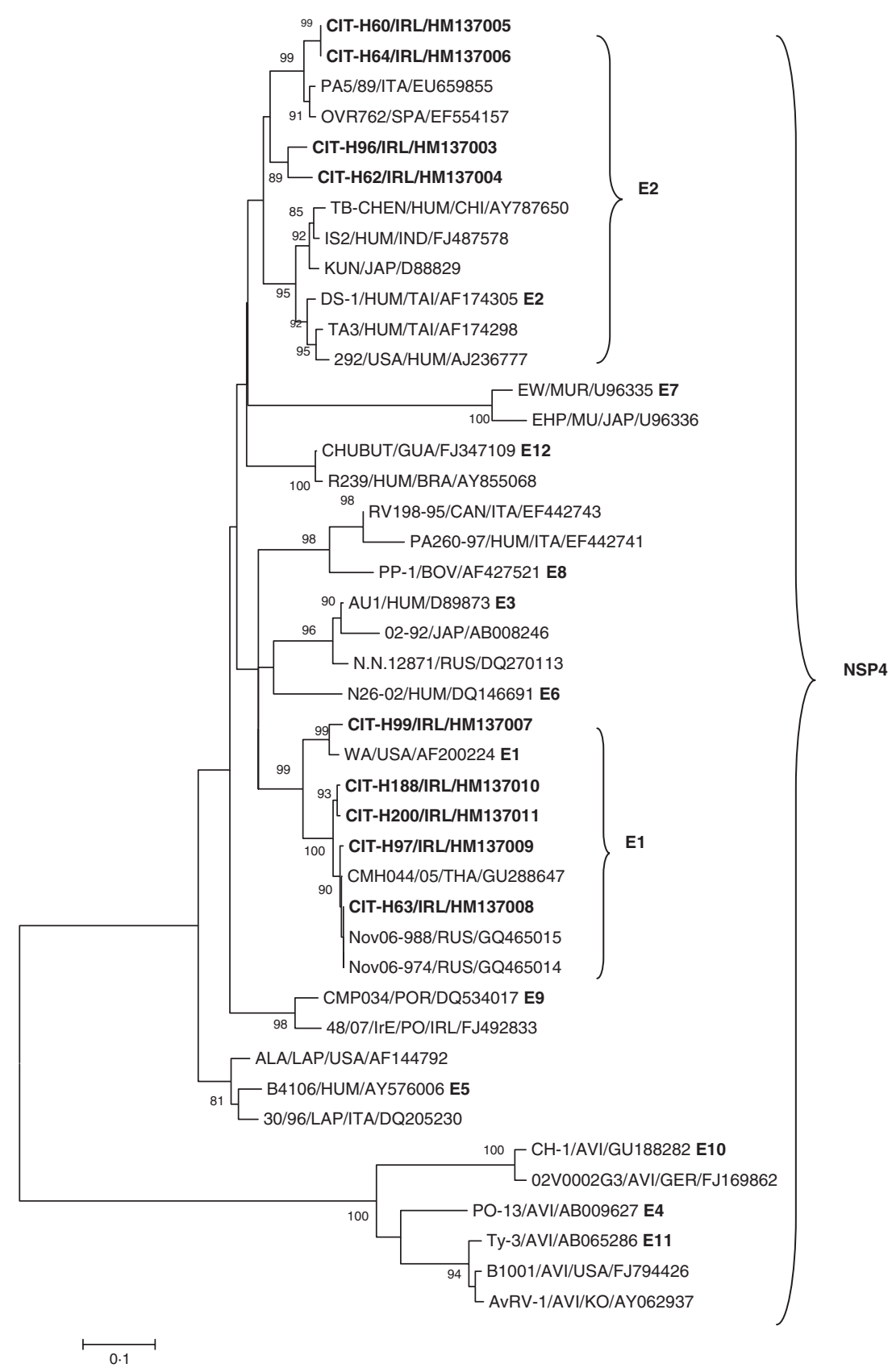

Fig. 5. Phylogenetic analysis of NSP4 nucleotide sequences, constructed using Clustal W alignment program and Mega4.0 (tree adapted from [7, 31]).

indicated that G1P[8], G3P[8], G2P[4], G9P[8], G1+ $\mathrm{G} 3 \mathrm{P}[8], \mathrm{G} 1+\mathrm{G} 2 \mathrm{P}[4+8]$ and $\mathrm{G} 1+\mathrm{G} 3+\mathrm{G} 4 \mathrm{P}[8]$ type combinations were present in Irish strains. The findings also highlighted the changes occurring in the circulating strains. G4 genotypes had previously been identified in the Irish population but were found to only be present as part of mixed infections in this study, although widely detected in other European countries [47].
Partial typing of rotavirus strains was also observed, in which the $G$ genotype was identified without the corresponding $\mathrm{P}$ genotype or vice versa, in some cases failure to amplify either gene (VP7 and VP4) was noted. The failure to amplify a RT-PCR product and subsequent typing of samples may be due to a number of issues relating to low viral load, possible presence of frame-shift mutations in the primer binding site, or the presence of a co-infecting virus 


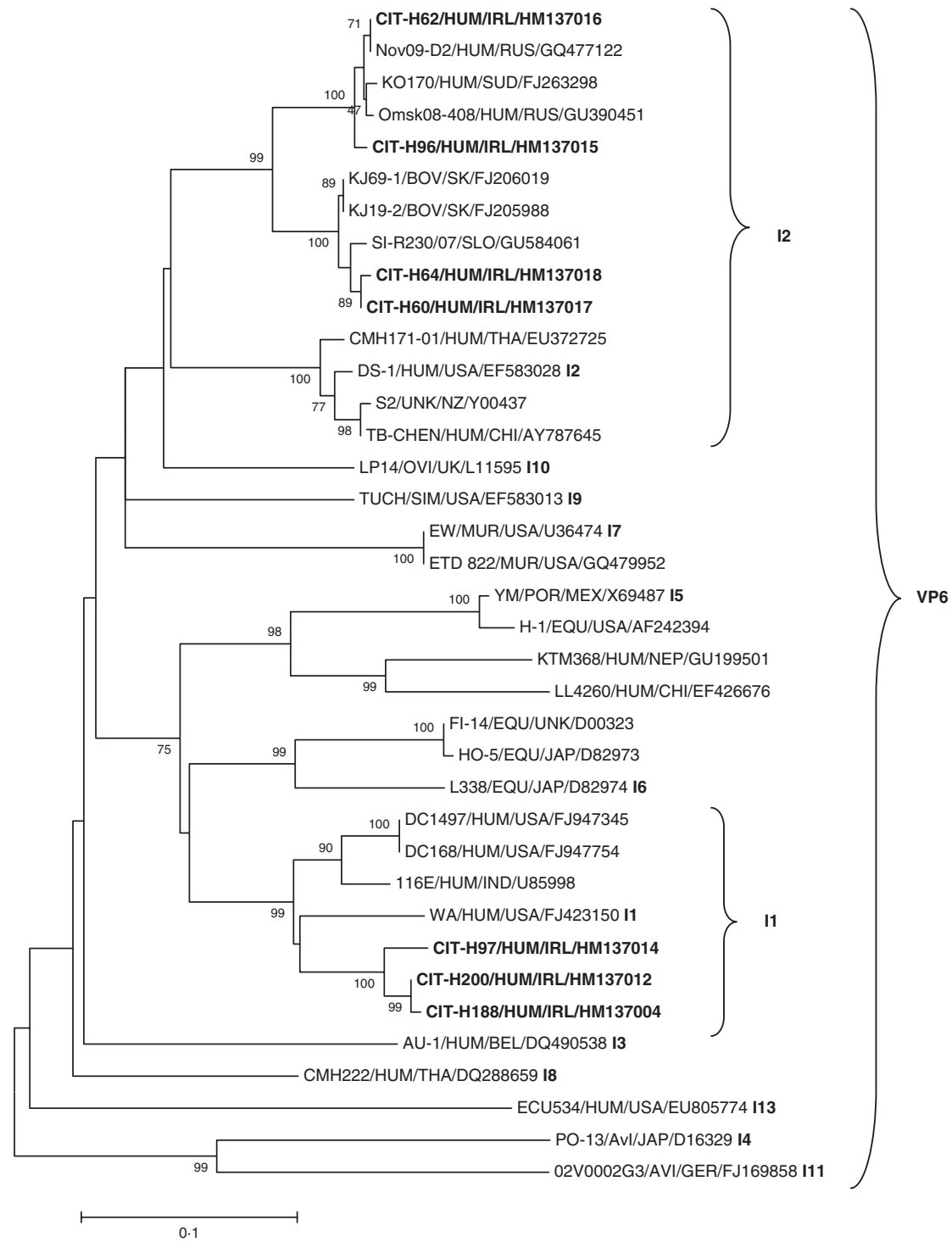

Fig. 6. Phylogenetic analysis of VP6 nucleotide sequences, constructed using Clustal W alignment program and Mega4.0 (tree adapted from [7]).

such as bocavirus which was identified in some unamplifiable samples (data not shown).

This study offers a clearer picture into the diversity of Irish rotavirus strains by not only highlighting the circulating strains, but by identifying the lineages from which these strains originate. Problems encountered in the last year of the study (2008-2009) resulted in lower sample numbers being available for analysis, these numbers are not a reflection of vaccinerelated efficacy or sampling bias merely technical issues in which restructuring in hospital practices meant samples were no longer available.

This is the first Irish study to report on NSP4 and VP6 gene segment-type combinations, the results of which mirror previously identified findings [31].

\section{NOTE}

Supplementary material accompanies this paper on the Journal's website (http://journals.cambridge.org/hyg).

\section{ACKNOWLEDGEMENTS}

Funding for this research was provided through the Food Institutional Research Measure (grant no. 05/ R\&D/CIT/365, awarded to H.O'S.), administered by the Department of Agriculture, Fisheries \& Food, Ireland. The authors thank Cork University Hospital, Mercy University Hospital, Bon Secours Hospital, Cork and Waterford Regional Hospital for their participation in this study. 


\section{DECLARATION OF INTEREST}

None.

\section{REFERENCES}

1. Parashar UD, et al. Global illness and death caused by rotavirus disease in children. Emerging Infectious Diseases 2003; 9: 565-572.

2. Estes MK, Kapikian AZ. Rotavirus. In: Knipe DM, et al., eds. Fields Virology, 5th edn, vol. 2. Philadelphia, PA: Lippincott-Raven, 2007, pp. 1917-1974.

3. Esona MD, et al. Reassortant group A rotavirus from straw-colored fruit bat (Eidolon helvum). Emerging Infectious Diseases 2010; 16: 1844-1852.

4. Collins PJ, et al. Identification of a G2-like porcine rotavirus bearing a novel VP4 type, P[32]. Journal of Veterinary Research 2010; 41: 41-73.

5. Gentsch JR, et al. Serotype diversity and reassortment between human and animal rotavirus strains: implications for rotavirus vaccine programs. Journal of Infectious Diseases 2005; 192: S146-59.

6. Santos N, Hoshino Y. Global distribution of rotavirus serotypes/genotypes and its implication for the development and implementation of an effective rotavirus vaccine. Reviews in Medical Virology 2005; 15: 29-56.

7. Matthijnssens J, et al. Recommendations for the classification of group A rotaviruses using all 11 genomic RNA segments. Archives of Virology 2008; 153: 1621-1629.

8. Kapikian AZ, Hoshino Y, Chanock RM. Rotaviruses. In: Knipe DM, et al., eds. Fields Virology, 4th edn. Lippincott Williams \& Wilkins, Philadelphia, PA, 2001, pp. 1787-1834.

9. Browne EP, Bellamy AR, Taylor JA. Membranedestabilizing activity of rotavirus NSP4 is mediated by a membrane-proximal amphipathic domain. Journal of General Virology 2000; 81: 1955-1959.

10. Matthijnssens J, et al. Are human $\mathrm{P}[14]$ rotavirus strains the result of interspecies transmissions from sheep or other ungulates that belong to the mammalian order Artiodactyla? Journal of Virology 2009; 83: 2917-2929.

11. Parez N. Rotavirus gastroenteritis: Why to back up the development of new vaccines? Comparative Immunology, Microbiology \& Infectious Diseases 2008; 31: 253-269.

12. Matthijnssens $\mathbf{J}$, et al. Molecular and biological characterization of the 5 human-bovine rotavirus (WC3)based reassortant strains of the pentavalent rotavirus vaccine, RotaTeq. Virology 2010; 403: 111-127.

13. Payne DC, et al. Rotavirus. United States-2008. Atlanta, GA, USA: Centre for Disease Control and Prevention, Manual for the Surveillance of VaccinePreventable Diseases, 2008, 4th edn, chapter 13 (http:// www.cdc.gov/vaccines/pubs/surv-manual/chpt13rotavirus.htm).

14. Vesikari T, et al. Efficacy of human rotavirus vaccine against rotavirus gastroenteritis during the first 2 years of life in European infants: randomised, double-blind controlled study. Lancet 2007; 370 1757-1763.

15. Centers for Disease Control. Delayed onset and diminished magnitude of rotavirus activity - United States. Morbidity and Mortality Weekly Report 2008; 57: 697-700.

16. O'Ryan M. Rotarix (RIX4414): an oral human rotavirus vaccine. Expert Review of Vaccines 2007; 6: 11-19.

17. Dennehy PH. Rotavirus vaccines: an overview. Clinical Microbiology Reviews 2008; 21 : 198-208.

18. Anon. Disease where vaccination might be recommended in the future. In: A Practical Guide to Immunisation. Chapter 4, pp. 49-50.

19. Barclay WS, Almond JW. Mutagensis of RNA virus genomes. In Cann AJ, ed. RNA Viruses: Practical Approach. Oxford University Press, 2000, pp. 26-29.

20. McHardy AC, Adams B. The role of genomics in tracking the evolution of influenza A virus. PLoS Pathogens 2009; 5: e1000566.

21. Skally M. Rotavirus in Ireland, 2007. Dublin: Ireland, Health Protection Surveillance Centre EPI-Insight, 2008, vol. 9, pp. 2-3.

22. O'Halloran F, et al. Molecular characterization of rotavirus in Ireland: detection of novel strains circulating in the population. Journal of Clinical Microbiology 2000; 38: 3370-3374.

23. Reidy N, et al. Emergence of G3 and G9 rotavirus and increased incidence of mixed infections in the southern region of Ireland. Journal of Medical Virology 2005; 77: 571-578.

24. Lennon G, et al. Changing profile of rotavirus in Ireland: predominance of $\mathrm{P}[8]$ and emergence of $\mathrm{P}[6]$ and $\mathrm{P}[9]$ in mixed infections. Journal of Medical Virology 2008; 80: 524-530.

25. Laemmli UK. Cleavage of structural proteins during the assembly of the head of bacteriophage. Nature 1970; 227: 680-685.

26. Gouvea V, et al. Polymerase chain reaction amplification and typing of rotavirus nucleic acid from stool specimens. Journal of Clinical Microbiology 1990; 28: 276-282.

27. Samajdar S, et al. Changing pattern of human group A rotaviruses: emergence of G12 as an important pathogen among children in eastern India. Journal of Clinical Virology 2006; 36: 183-188.

28. Gentsch JR, et al. Identification of group A rotavirus gene 4 types by polymerase chain reaction. Journal of Clinical Microbiology 1992; 30: 1365-1373.

29. Simmonds MK, et al. New oligonucleotide primers for P-typing of rotavirus strains: Strategies for typing previously untypeable strains. Journal of Clinical Virology 2008; 42: 368-373.

30. Iturriza-Gómara M, et al. Diversity within the VP4 gene of rotavirus $\mathrm{P}[8]$ strains: implications for reverse transcription-PCR genotyping. Journal of Clinical Microbiology 2000; 38: 898-901.

31. Bányai K, et al. Assignment of the group A rotavirus NSP4 gene into genotypes using a hemi-nested multiplex PCR assay: a rapid and reproducible assay for strain 
surveillance studies. Journal of Medical Microbiology 2009; 58: 303-311.

32. Iturriza Gómara M, et al. Rotavirus subgroup characterisation by restriction endonuclease digestion of a cDNA fragment of the VP6 gene. Journal of Virological Methods 2002; 105: 99-103.

33. UNICEF/World Health Organisation. Diarrhoea: why children are still dying and what can be done. NLM classification: WS 312, 2009.

34. Taniguchi K, et al. Nucleotide sequence of VP4 and VP7 genes of human rotaviruses with subgroup I specificity and long RNA pattern: implication for new $\mathrm{G}$ serotype specificity. Journal of Virology 1990; 64: 5640-5644.

35. Bányai K, et al. Emergence of serotype G12 rotaviruses, Hungary. Emerging Infectious Diseases 2007; 13: 916-919.

36. Pongsuwanna Y, et al. Detection of a human rotavirus with G12 and P[9] specificity in Thailand. Journal of Clinical Microbiology 2002; 40: 1390-1394.

37. Das S, et al. Emergence of novel human group A rotavirus G12 strains in India. Journal of Clinical Microbiology 2003; 41 : 2760-2762.

38. Castello AA, et al. Characterization of genotype P[9]G12 rotavirus strains from Argentina: high similarity with Japanese and Korean G12 strains. Journal of Medical Virology 2009; 81: 371-381.

39. Matthijnssens J, et al. Phylodynamic analyses of rotavirus genotypes G9 and G12 underscore their potential for swift global spread. Molecular Biology and Evolution 2010; 27 : 2431-2436.

40. Bok K, et al. Emergence of G9 P[6] human rotaviruses in Argentina: phylogenetic relationships among G9 strains. Journal of Clinical Microbiology 2001; 39: 4020-4025.

41. Martella V, et al. Detection of the emerging rotavirus G9 serotype at high frequency in Italy. Journal of Clinical Microbiology 2003; 41: 3960-3963.

42. Khamrin P, et al. Emergence of human G9 rotavirus with an exceptionally high frequency in children admitted to hospital with diarrhea in Chiang Mai, Thailand. Journal of Medical Virology 2006; 78: 273-280.

43. Rubilar-Abreu E, et al. Serotype G9 rotavirus infections in adults in Sweden. Journal of Clinical Microbiology 2005; 43: 1374-1376.

44. Feeney SA, et al. Association of the G4 rotavirus genotype with gastroenteritis in adults. Journal of Medical Virology 2006; 78: 1119-1123.

45. Cilla G, et al. Rotavirus genotypes in children in the Basque country (northern Spain) over a 13-year period (July 1996-June 2009). European Journal of Clinical Microbiology \& Infectious Diseases 2010; 29: 955-960.

46. Yang SH, et al. Molecular epidemiology of rotavirus among children under 5 years old hospitalized for diarrhea in China. Chinese Journal of Experimental and Clinical Virology 2009; 23: 168-170.

47. Iturriza-Gómara M, et al. Rotavirus genotypes cocirculating in Europe between 2006 and 2009 as determined by EuroRotaNet, a pan-European collaborative strain surveillance network. Epidemiology and Infection. Published online: 16 August 2010. doi:10.1017/S0950268810001810.

48. Cunliffe NA, et al. Rotavirus strain diversity in Blantyre, Malawi, from 1997 to 1999. Journal of Clinical Microbiology 2001; 39: 836-843.

49. Samajdar S, et al. Human group A rotavirus $\mathrm{P}[8]$ Hun9-like and rare OP354-like strains are circulating among diarrhoeic children in Eastern India. Archives of Virology 2008; 153: 1933-1936.

50. Theamboonlers A, et al. Molecular characterization of group A human rotaviruses in Bangkok and Buriram, Thailand during 2004-2006 reveals the predominance of G1P[8], G9P[8] and a rare G3P[19] strain. Virus Genes 2008; 36: 289-298.

51. Nguyen TA, et al. Use of sequence analysis of the VP4 gene to classify recent Vietnamese rotavirus isolates. Clinical Microbiology and Infection 2008; 14: 235-241.

52. Nagashima S, et al. Characterization of full-length VP4 genes of OP354-like P[8] human rotavirus strains detected in Bangladesh representing a novel $\mathrm{P}[8]$ subtype. Archives of Virology 2009; 154: 1223-1231.

53. Nagashima S, et al. Identification of $\mathrm{P}[8] \mathrm{b}$ subtype in OP354-like human rotavirus strains by a modified RT-PCR method. Japanese Journal of Infectious Diseases 2010; 63: 208-211.

54. Cashman O, et al. Changing profile of the bovine rotavirus G6 population in the south of Ireland from 2002-2009. Journal of Veterinary Microbiology 2010; 146: $238-244$.

55. Tamura K, et al. Mega 4: Molecular evolutionary genetics analysis (MEGA) software version 4.0. Molecular Biology and Evolution 2007; 24: 1596-1599.

56. Phan TG, et al. Evidence of intragenic recombination in G1 Rotavirus VP7 genes. Journal of Virology 2007; 81 : 10188-10194.

57. Martinez-Laso J, et al. Diversity of the G3 genes of human rotaviruses in isolates from Spain from 2004 to 2006: cross-species transmission and inter-genotype recombination generates alleles. Journal of General Virology 2009; 90: 935-943.

58. Phan TG, et al. Genetic Heterogeneity, evolution and recombination in emerging G9 rotaviruses. Infection, Genetics and Evolution 2007; 7: 656-663.

59. Espínola EE, et al. Sequence and phylogenetic analysis of the VP4 gene of human rotaviruses isolated in Paraguay. Archives of Virology 2008; 153: 1067-1073. 